\title{
Design of a Hydrogen Compressor Powered by Accumulated Heat and Generated in Metal Hydrides
}

\author{
Natália Jasminská ${ }^{1}$, Tomáš Brestovič² L Lubica Bednárová $^{3}$, Marián Lázár ${ }^{4}$, \\ Romana Dobáková ${ }^{5}$
}

Department of Power Engineering, Faculty of Mechanical Engineering, Technical University of Košice, 04200 Košice, Slovak Republic

\begin{abstract}
The proposed article describes a compressor design for using the heat generated in the hydrogen absorption process into the metal alloy during compression. The absorption of hydrogen into the alloy occurs after the desired pressure has been achieved, whereby the catalytic effect of the used metals to dissociate the hydrogen molecule and the subsequent diffusion of the hydrogen atoms into the intermetallic space of the lattice occur. The absorption process is accompanied by a gradual increase in pressure up to the total saturation value of the metal. The equilibrium pressure at which the absorption occurs is highly dependent on the temperature of the alloy. The difference in the equilibrium pressures of MH materials at an acceptable temperature change has led to efforts of creating a hydrogen compressor that would use MH heating and refrigeration heat cycle.
\end{abstract}

Keywords—hydrogen, alloy, compressor, compression, absorption.

\section{INTRODUCTION}

In nature hydrogen does not naturally occur in an unbound molecular form. It is most often produced through the partial oxidation of natural gas, steam reforming and electrolysis of water.

If it is not in use immediately after production, it is necessary to store it. Most storage methods, in view of the low hydrogen density, require the use of a compressor that increases the pressure to the desired level.

The hydrogen compressor is currently used to increase hydrogen pressure mainly through mechanical means. Rotary turbines are used at high volumetric flow rates, and at low flow rates there are mainly used piston compressors, or pressure multipliers utilizing hydraulic mechanisms. There was an attempt to test compressors using proton exchange membranes and simple compressors with metal hydride alloy.

The compressor powered by accumulated heat and generated in metal hydrides operates in a closed thermodynamic cycle, where hydrogen is stored at low temperatures with the catalytic effect of metals. Then the hydrogen molecules are dissociated and atomic diffusion into the intermetallic structure of the alloy occur. Once the alloy is saturated with hydrogen, it is necessary to bring in heat from an external source, thus increasing the pressure by shifting the working point to a higher isothermal curve. At higher temperatures, it is possible to desorb hydrogen from the alloy and reduce the storage capacity to the original state. It is possible to shift the subsequent lowering of the temperature to the starting point [1-4].

\section{The BASIC Characteristics OF A Hydrogen COMPRESSOR}

The compressor for compressing hydrogen, which is driven by the interaction of hydride formation in a metal or intermetallic compound with hydrogen to the metal hydride, is considered to be a promising application for the hydrogen energy system. The advantage of a hydrogen compressor rests in its simplicity in the design, compactness, safety, reliability, absence of moving parts and the possibility of consuming waste heat instead of electricity.

$$
\mathrm{M}(\mathrm{s})+\frac{\mathrm{x}}{2} \mathrm{H}_{2}(\mathrm{~g}) \stackrel{\text { absorption } / \text { desorption }}{\longrightarrow} \mathrm{MH}_{\mathrm{x}}(\mathrm{s})+\mathrm{Q}
$$

where: M-metals or alloys, with a solid phase, $\mathrm{g}$ - gas phase, Q- temperature generated during absorption (J).

The process of forming the metal hydride by absorbing hydrogen into the metal alloy is accompanied by heat release Q. In desorption process, that is, during decomposition, the alloy consumes approximately the same amount of heat. 
The absorption and desorption process is strongly dependent on the proper characteristic of the reaction (1), including its thermodynamic and kinetic characteristics, including the very important heat and mass transfer. Important aspects also include the composition, structure and morphology of the solid phase connected to the process. These properties are mainly related to the basic aspects of the studied metal hydride materials.

The use of metallic hydrides for the thermal compression of hydrogen is based on the equilibrium pressure peq, the equilibrium pressure change and the temperature described by the van Hoff equation [5, 6]:

$$
\ln p_{\text {eq }}=\frac{\Delta H}{R T}-\frac{\Delta S}{R}
$$

Where $\Delta H$ - the enthalpy change $\left(\mathrm{J} \cdot \mathrm{mol}^{-1}\right), \Delta S$ - change of entropy connected with the absorption or desorption in a metal hydride $\left(\mathrm{J} \cdot \mathrm{mol}^{-1} \cdot \mathrm{K}^{-1}\right)$.

The compressor starts performing thedesorption of hydrogen if the equilibrium pressure of the hydride exceeds the set pressure value. In this case, the storage pressure is constant with the set pressure value. The operating principle of the singlelevel hydrogen compressor is illustrated in Fig. 1.

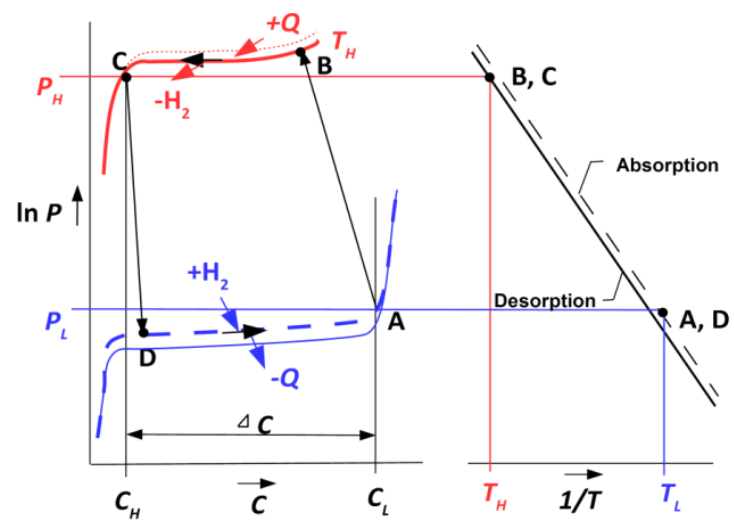

FIGURE 1: The operating principle of the single-level MHx hydrogen compressor [6]

As shown in Fig. 1, the operation of the single-level compressor consists of 4 processes:

DA - hydrogen absorption in low temperature (TL) and low pressure (PL),

$\mathrm{AB}$ - adequate heating and compression when changing from TL to TH temperature,

$\mathrm{BC}$ - the desorption of compressed hydrogen at the $\mathrm{TH}$ temperature and elevated $\mathrm{PH}$ pressure,

$\mathrm{CD}$ - adequate cooling from TH to TL.

According to the equation (2) the pressure increases exponentially with the increasing temperature and large pressure values can be obtained through slight temperature changes. On the basis of the metal hydride the compressor can be designed to cover a wide range of operating pressure ratios using suitable alloys.

To achieve high pressure, the individual units of the hydride can be connected in a series, each unit containing a different kind of alloy and consequently increasing pressures. The most important properties of a suitable alloy for hydrogen compression are: good hydrogen absorption - desorption rate, smaller enthalpy change, rapid reaction kinetics and good structural stability during cycles. High pressure gradient metal hydrides are suitable for the compressor depending on the temperature change, especially in a low temperature area. The efficiency of the single-level hydrogen compressor is given by the relation [6]:

$$
\eta=\frac{m \cdot\left(R \cdot T_{\mathrm{H}} \cdot \ln p_{\mathrm{H}}-R \cdot T_{\mathrm{L}} \cdot \ln p_{\mathrm{L}}\right)}{m \cdot c_{\mathrm{p}} \cdot\left(T_{\mathrm{H}}-T_{\mathrm{L}}\right)+\Delta H}
$$

where: $\eta$ is the efficiency of (-), $m$ - the weight of the metal hydride $(\mathrm{kg}), c_{\mathrm{p}}$ - the specific heat capacity of the metal hydride $\left(\mathrm{J} \cdot \mathrm{kg}^{-1} \cdot \mathrm{K}^{-1}\right), \quad p_{\mathrm{L}^{-}}$equilibrium pressure at the absorption temperature $T_{\mathrm{L}}(\mathrm{Pa}), p_{\mathrm{H}}$ - equilibrium pressure at the 
desorptiontemperature $T_{\mathrm{H}}(\mathrm{Pa}), \Delta H$ - the enthalpy change of the metal hydride $\left(\mathrm{kJ} \cdot \mathrm{mol}^{-1}\right), R$ - universal gas constant $\left(\mathrm{J} \cdot \mathrm{mol}^{-}\right.$ $\left.{ }^{1} \cdot \mathrm{K}^{-1}\right)$.

The hydrogen absorption and desorption kinetics are significantly influenced by the thermal conductivity and porosity of various metal hydrides. The hydrogen is first absorbed into the metal hydride at a low pressure and temperature [7, 8]. The hydrogen remains in the hydride unless exposed to an increased temperature or pressure drop. In the case that the temperature increase is sufficient and the pressure of the high-pressure vessel is less than the pressure in the MH tank, the hydrogen leaves the $\mathrm{MH}$ tank at pressures ranging from 3 to 10 times the original fed pressure. Multi-level metal hydride hydrogen compressors use a combination of different types of metal hydrides to increase the overall compression ratio. A two-level metal hydride compressor is shown in Fig. 2 [9].

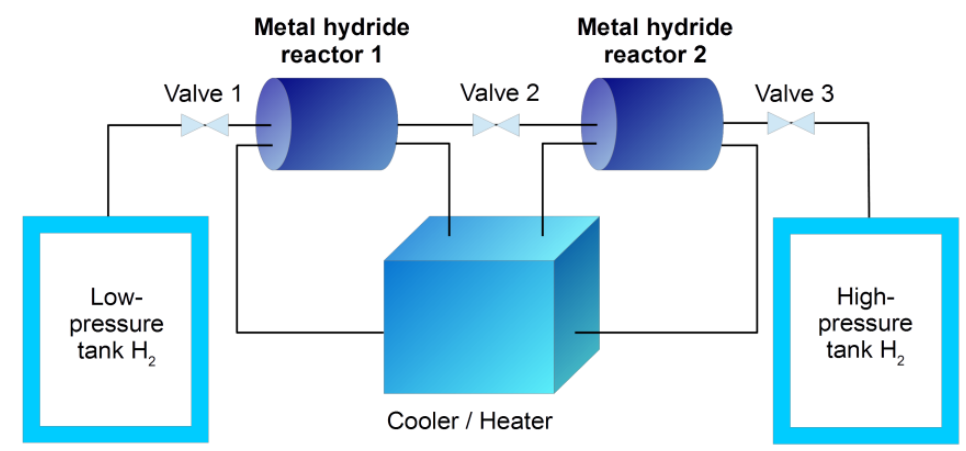

FIGURE 2: A diagram of a two-level metal hydride compressor

The concept of a multi-level compressor is based on the compression of a single level. It has been shown that by increasing the input pressures, the compression ratio remains almost constant. Using a two-level system, it is possible to increase the final pressure in the system using the second compression cycle [9].

\section{PROPOSAL FOR A HYDROGEN COMPRESSOR}

With compressors using metal hydride alloys there is a significant transport and heat management mechanism. The absorption of hydrogen into the metal alloy results in the release of heat, which proportionally increases the equilibrium gas pressure. The absorption of hydrogen at a relatively lower temperature, ensured by cooling, occurs at a lower pressure.

After the absorption and subsequent heat transfer, a significant increase in hydrogen pressure occurs, with a compression ratio of more than 3, with a temperature increase by several tenths of degrees. Increasing the kinetics process is achieved through the proper design of the pressure vessel and the intensification of the heat inlet and outlet. A new, original proposed solution to ensure continuous operation is the tandem connection of the metal hydride tanks with the heat-exchange provided with a heat pump (Fig. 3).

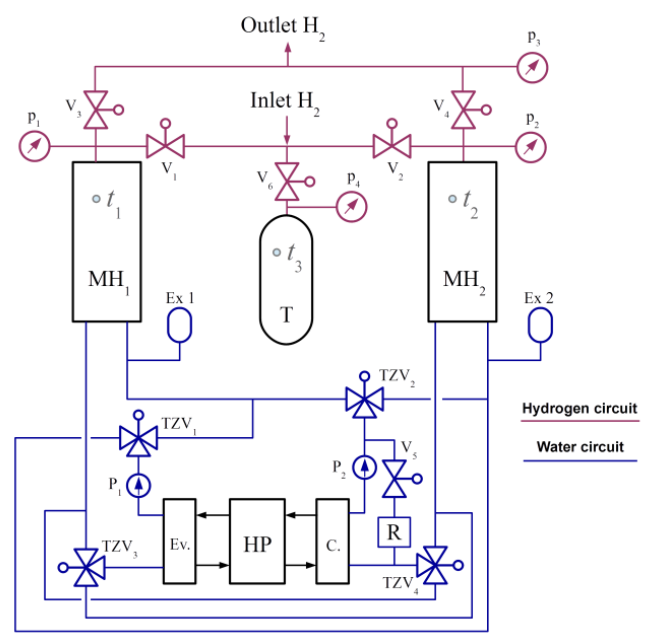

FIGURE 3: Tandem hydrogen compressor block diagram 
The essence of the development is to connect the heat pump (HP) and tandem arrangement of the metal hydride tanks (MH and $\mathrm{MH}_{2}$ ) to continuous hydrogen compression by using the thermal cycle. The heat pump removes the heat from the storage tank where the hydrogen is absorbed and transported into a tank where the hydrogen is already absorbed to increase the pressure. After compressing and releasing the hydrogen into the end pressure vessel, a reverse heat transfer process occurs between the tanks to ensure the continuous operation of the compressor. The advantage of the solution is the continuous and safe compression of the hydrogen flow volumes. Safety is ensured by preventing the contact of system's moving parts with the compressed hydrogen. A pressure vessel (T) that temporarily stores hydrogen on the low pressure side serves to ensure continuous operation even during the adsorption/desorption cycle. To remove excess heat on the side of the condenser $(\mathrm{C}$.), an additional cooler $(\mathrm{R})$ serves.

The preparation and composition of the metal hydride alloy is the key to its storage properties. The working pressure of the compressor level is dependent on the composition of the alloy. It is also necessary to define the degradation of the metal hydride alloy in the use of hydrogen with the oxygen admixture produced during the electrolysis of water.

\section{SElEction OF A Suitable TyPe OF A Metal HydRIDE FOR HydROGEN COMPRESSOR}

The selection of the hydrogen compressor tanks is influenced by several parameters such as: tank capacity, alloy absorption capacity, minimum filling time, maximum discharge period, maximum pressure range, temperature range and the composition of the alloy that fills the tank. The HBond 500 metal hydride tank was used to design the hydrogen compressor. The nominal capacity of the metal hydride tank is $0.5 \mathrm{~m}^{3} \mathrm{H}_{2}$. The tank is produced from stainless steel and is equipped with an internal heat exchanger, an internal filter and a quick connector for connecting hydrogen.

The HBond 500 can be filled with hydrides with different thermodynamic properties. The test pressure is 30 bar. The used container is filled with basic type AB5 alloy with alloy composition $\mathrm{La}_{0.85} \mathrm{Ce}_{0.15} \mathrm{Ni}_{5}$.

The maximum pressure in filling the tank is 15 bar. The maximum inlet hydrogen temperature can reach $40{ }^{\circ} \mathrm{C}$. The hydrogen tank is provided with an internal heat exchanger as shown in Fig. 4.

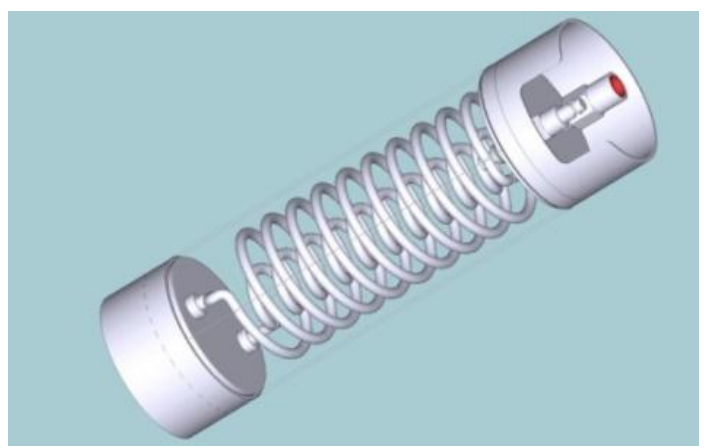

FIGURE 4: H Bond 500 Tank

The rate of hydrogen desorption is dependent on the temperature of the metal hydride. The maximum tank discharge rate is $8.33 \mathrm{Nl} \cdot \mathrm{min}^{-1}$. The reaction heat is $1 \mathrm{MJ} \cdot \mathrm{m}^{-3}$ of the desorbed hydrogen and this heat must be supplied through a water circuit.

The metal alloy contained in the tanks was tested in the Material Science Institute at Helsinki Technical University, where no degradation of the absorption capacity was observed even after 1000 cycles. The minimum purity of hydrogen is $99.9 \%$. Since the metal hydride does not release any other impurities, the purity of the hydrogen at the outlet is the same or even better than the inlet.

The storage system is equipped with all the tools and valves necessary for its proper function throughout the entire system.

Experimental measurements for the setting of pressure and temperature dependence for the creation of P-T characteristics were performed on the alloy $\mathrm{La}_{0.85} \mathrm{Ce}_{0.15} \mathrm{Ni}_{5}$. The obtained data will serve the primary thermodynamic design of the hydrogen compressor.

Fig. 5 is a $p-T$ diagram depicting the pressure dependence on the concentration of stored hydrogen in isothermal conditions. The measurement of the pressure-to-temperature dependence was performed at a constant concentration of hydrogen $(0.9$ wt.\%). The measurement of the absorption capacities of metal hydride alloys and pressure gradients will be performed continuously with the formation of new types of alloys. 


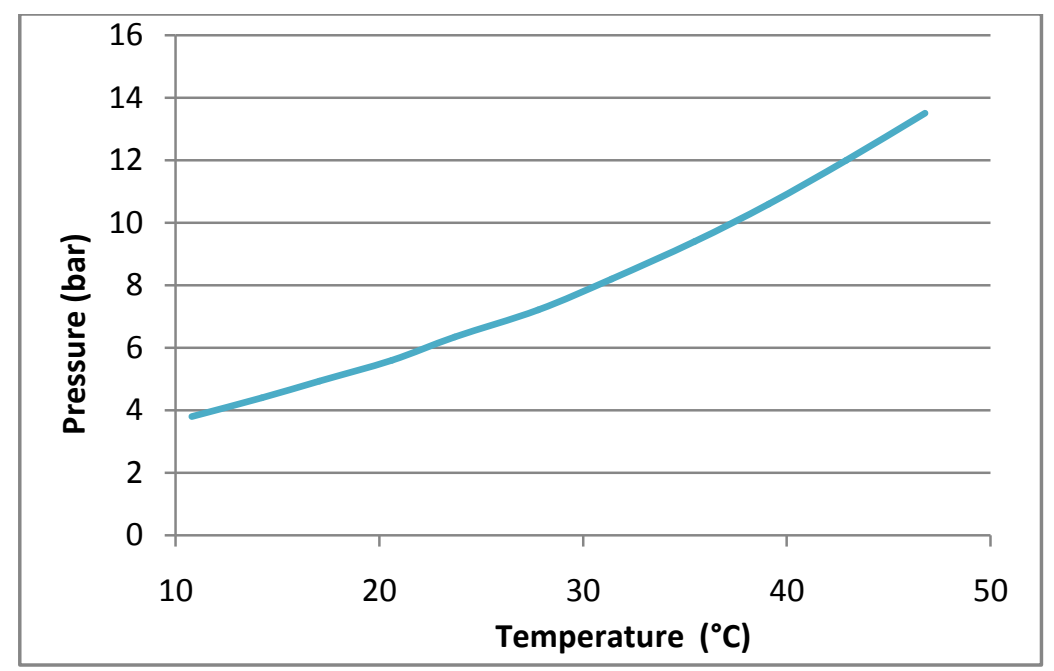

FIGURE 5: P-T characteristic of alloy $\mathrm{La}_{0.85} \mathrm{Ce}_{0.15} \mathrm{Ni}_{5}$ at $0.9 \mathrm{wt} \%$ of the stored hydrogen

\section{CONCLUSION}

The article described the basic concept of the development of a hydrogen compressor using metal hydride materials with the tandem connection of tanks. A heat pump is designed for heat transfer, which minimizes the energy demand of hydrogen compression.

The development of a hydrogen compressor has great potential for innovative social and economic needs in the development and application of hydrogen technologies in the automotive industry and transport, mainly in the context of Slovak and European innovation strategy.

The economic benefit of the submitted work is limited by the implementation of the hydrogen economy, mainly in the automotive industry in Slovakia. The hydrogen compressor prototype is a unique solution in relation to safety of operation, since hydrogen does not come in contact with moving or compressing elements during compression or with the rotating parts of the system.

\section{ACKNOWLEDGEMENTS}

This paper was written with the financial support of the granting agency APPV within the project solution No. APVV-150202, of the granting agency VEGA within the project solution No. 1/0752/16 and of the granting agency KEGA within the project solution No. 005TUKE-4/2016.

\section{REFERENCES}

[1] S. Mellouli, F. Askri, H. Dhaou, A. Jemni, S. Ben Nasrallah, "A study of the thermal behavior of a deformable metal-hydride bed," International journal of hydrogen energy, pages 1711-1724. November 2006.

[2] S. Mellouli, F. Askri, H. Dhaou, A. Jemni, S. Ben Nasrallah, "Hydrogen storage in metal hydride tanks equipped with metal foam heat exchanger". International journal of hydrogen energy, pages 9393 - 9401. October 2009.

[3] B.SIKINTUNA, F.LAMARI-DARKRIM, M. HIRSCHER, "Metal hydride materials for solid hydrogen storage: A Review," International journal of hydrogen energy, pages 1121 - 1140. November 2006.

[4] Z.Cao, L. Ouyang,H.Wang, J. Liu, D.Sun, Q.Zhang, M.Zhu, “Advanced high-pressure metal hydride fabricated via TieCreMn alloys for hybrid tank," International journal of hydrogen energy, pages 2717 - 2728. December 2014

[5] M.V.Lototsky,V.A.Yartys,B.G.Pollet,Jr.R.C,Boweman, "Metal hydride hydrogen compressors: A review.," International journal of hydrogen energy, pages: 5818 - 5851. January 2014.

[6] G.Popeneciu, V.Almasan, Coldea, D. I. Lupu,I.Misan, O.Ardelean, "Investigation on a tree-stage hydrogen thermal compressor based on metal hydrides,” Journal of Physics: Conference Series 182, 012053. 2009.

[7] A. Sarkar,R.Benerjee, "Net energy analysis of hydrogen storage option," International journal of hydrogen energy, pages: 867-877. 2005.

[8] R.Schulz, J.Huot,G.Liang, S.Boily,G.Lalande, MC. Denis,etal.,"Recent development in the applications of nanocrystalline materials to hydrogen technologies, “ Mater SciEng A 1999;267:240

[9] M.Hirscher, M.Becher, "Hydrogen storage in carbon nanotubes," J Nanosci Nanotech 2003; 3(1-2):3-17. 